\title{
ОПТИМАЛЬНЫЙ СИНТЕЗ \\ ИНТЕРФЕРЕНЦИОННО-ПОЛЯРИЗАЦИОННЫХ СВЕТОФИЛЬТРОВ
}

\section{Введение}

В предыдущей статье $\left[{ }^{1}\right]$ были выведены формулы, определяющие коэффициент пропускания интерференционно-поляризационного светофильтра типа Шольца в зависимости от длины волны. В настоящей статье применим эти формулы к синтезу светофильтров, обладающих оптимальной кривой пропускания. Примем толщины всех двупреломляющих пластинок равными; тогда задача оптимизации сведется к нахождению наиболее выгодных азимутов пластинок. В качестве критерия оптимальности применим принцип интегральной минимизации коэффициента пропускания вне полосы пропускания. Аналогичные критерии были ранее широко использованы в различных задачах теории интерференционных пленок (см. $\left.\left[{ }^{2}\right]\right)$.

\section{Исходные формулы}

Если интерференционно-поляризационный светофильтр типа Шольца состоит из двупреломляющих пластинок равной толщины, то элементы матрицы $F$, преобразующей матрицу поляризации проходящего сквозь фильтр света, даются, согласно формулам (15), (16), (27) и (28) статьи $\left[{ }^{1}\right]$, следующими выражениями:

$F_{21}=-F_{12}^{*}=\sum_{s=0,1, \ldots, N} i^{s} \sin ^{s} \gamma \cos ^{N-s} \gamma \sin \left(2 \varphi_{k_{s}}-2 \varphi_{k_{s-1}}+\ldots \ldots-(-1)^{s} 2 \varphi_{k_{1}}\right)$,

$F_{11}=F_{22}^{*}=\sum_{s=0,1, \ldots, N} i^{8} \sin ^{s} \gamma \cos ^{N-s} \gamma \cos \left(2 \varphi_{k_{s}}-2 \varphi_{k_{s-1}}+\ldots \ldots-(-1)^{s} 2 \varphi_{k_{1}}\right)$

или

$$
F_{21}=-F_{12}^{*}=
$$

$=\sum_{s=1,3, \ldots} i^{s+1} \mathrm{x} \varphi_{N+1, N} \mathrm{X} \varphi_{N, N-1} \ldots \mathrm{x} \varphi_{10} \exp \left[-i \gamma\left(\varepsilon_{N}+\varepsilon_{N-1}+\ldots+\varepsilon_{1}\right)\right]$,

$F_{11}=F_{22}^{*}=\sum_{s=0,2, \ldots} i^{s} \times \varphi_{N+1, N} \times \varphi_{N, N-1} \ldots \times \varphi_{10} \exp \left[i \gamma\left(\varepsilon_{N}+\varepsilon_{N-1}+\ldots+\varepsilon_{1}\right]\right.$.

В этих формулах $N-$ число пластинок,

$$
\gamma=(k h / 2)\left(n_{o}-n_{e}\right),
$$

где $n_{o}, n_{e}$ - показатели преломления обыкновенного и необыкновен- 
ного лучей, $h$ - толщина одной пластинки и $k-$ волновое число. В формулах (1) и (2) каждому значению индекса $s$ соответствует $\frac{N !}{s !(N-s) !}$ членов суммы, отличающихся комбинациями значений индексов $k_{s}, k_{s-1}, \ldots, k_{1}$ (причем $N \geqslant k_{s}>k_{s-1}>\ldots>k_{1} \geqslant 1$ ); $\varphi_{1}, \varphi_{2}, \ldots, \varphi_{N}$ означают азимуты пластинок (нумерация идет в направлении падения света). Всего в суммах (1) и (2) по $2^{N}$ членов. В формулах (3) и (4)

$$
\begin{aligned}
\varphi_{k+1, k} & =\varphi_{k+1}-\varphi_{k}, \\
\varphi_{N+1} & =\varphi_{0}=0 ;
\end{aligned}
$$

х означает синус или косинус; $s$ есть число синусов среди х'ов; каждому значению $s$ соответствует $\frac{(N+1) !}{s !(N+1-s) !}$ членов суммы, отличающихся комбинациями значений индексов аргументов синусов; $\varepsilon_{N}, \varepsilon_{N-1}, \ldots, \varepsilon_{1}$ равны +1 или -1 согласно правилам: $\varepsilon_{N}=+1$, если х $\varphi_{N+1, N}$ есть косинус, и $\varepsilon_{N}=-1$, если х $\varphi_{N+1, N}$ есть синус; $\varepsilon_{k} \varepsilon_{k-1}=+1$, если х $\varphi_{k, k-1}$ есть. косинус, и $\varepsilon_{k} \varepsilon_{k-1}=-1$, если х $\varphi_{k, k-1}$ есть синус; множитель х $\varphi_{10}$ не участвует в определении знаков $\varepsilon$, но в каждом члене он является синусом или косинусом с тем, чтобы общее число синусов было в членах суммы (3) нечетным, а в членах суммы (4) четным. Всего в этих суммах по $2^{N}$ членов.

Перепишем формулы (3) и (4) в виде:

$$
F_{21}=-F_{12}^{*}=e^{-i N \gamma}\left(a_{0}+a_{1} e^{2 i \nu}+\ldots+a_{N} e^{2 i N \gamma}\right)
$$

и

$$
F_{11}=F_{22}^{*}=e^{-i N \gamma}\left(b_{0}+b_{1} e^{2 i \gamma}+\ldots+b_{N} e^{2 i N \gamma}\right),
$$

где коэффициенты $a_{0}, a_{1}, \ldots, a_{N}$ и $b_{0}, b_{1}, \ldots, b_{N}$ вещественны и зависят определенным образом только от азимутов $\varphi_{1}, \varphi_{2}, \ldots, \varphi_{N}$ пластинок. Из этих формул следует:

$$
\begin{aligned}
& F_{21} F_{21}^{*}=A_{0}+A_{1} \cos ^{2} \gamma+\ldots+A_{N} \cos ^{2 N} \gamma, \\
& F_{11} F_{11}^{*}=B_{0}+B_{1} \cos ^{2} \gamma+\ldots+B_{N} \cos ^{2 N} \gamma,
\end{aligned}
$$

где $A_{0}, A_{1}, \ldots, A_{N}$ выражаются в виде квадратичных форм через $a_{0}, a_{1}, \ldots, a_{N}$, а $B_{0}, B_{1}, \ldots, B_{N}-$ в виде таких же квадратичных форм через $b_{0}, b_{1}, \ldots, b_{N}$. При этом, так как

$$
\|F\|=F_{11} F_{11}^{*}+F_{21} F_{21}^{*}=1 \text {, }
$$

To

$$
\begin{aligned}
& B_{0}=1-A_{0}, \\
& B_{k}=-A_{k}, \quad k=1,2, \ldots, N .
\end{aligned}
$$

Согласно формулам (29) и (30) статьи [ $\left.{ }^{1}\right]$, коэффициент пропускания фильтра равен или $F_{11} F_{11}^{*}$, или $F_{21} F_{21}^{*}$, в зависимости от ориентаций входного и выходного поляризаторов: в первом случае поляризаторы параллельны, во втором скрещены. Оба случая, в силу симметрии формул (9), (10) и (12), вполне аналогичны друг другу. Поэтому ограничимся в нижеследующем рассмотрением только второго случая. Коэффициент пропускания $I_{N}$ равен тогда

$$
I_{N}(\cos \gamma)=F_{21} F_{21}^{*}=A_{0}+A_{1} \cos ^{2} \gamma+\ldots+A_{N} \cos ^{2 N} \gamma .
$$

Таковы основные исходные формулы. Вдобавок приведем еще ряд 
дополнительных формул, которые будут нам нужны в процедуре синтеза.

Коэффициенты $a_{k}, b_{k}, A_{k}$ и $B_{k}(k=0,1, \ldots, N)$ удовлетворяют некоторым простым соотношениям. Положив в формулах (1) и (2) $\cos \gamma= \pm 1$, получим $F_{11}=( \pm 1)^{N}$ и $F_{21}=0$. Отсюда, согласно формулам (7)-(10), имеем:

и

$$
a_{0}+a_{1}+\ldots+a_{N}=A_{0}+A_{1}+\ldots+A_{N}=0
$$

$$
b_{0}+b_{1}+\ldots+b_{N}=B_{0}+B_{1}+\ldots+B_{N}=1 \text {. }
$$

Эти соотношения удовлетворяются тождественно, независимо от азимутов пластинок. Из формул (13) и (14) вытекает также, что

$$
I_{N}( \pm 1)=0 \text {. }
$$

Положим, далее, в формулах (1), (2), (7) и (8) $\cos \gamma=0, \sin \gamma= \pm 1$. В результате получим соотношения:

$$
\begin{aligned}
(\mp i)^{N} F_{21} & =\sin \left(2 \varphi_{N}-2 \varphi_{N-1}+\ldots-(-1)^{N} 2 \varphi_{1}\right)= \\
& =(-1)^{N}\left(a_{0}-a_{1}+-\ldots+(-1)^{N} a_{N}\right), \\
(\mp i)^{N} F_{11} & =\cos \left(2 \varphi_{N}-2 \varphi_{N-1}+\ldots \ldots-(-1)^{N} 2 \varphi_{1}\right)= \\
& =(-1)^{N}\left(b_{0}-b_{1}+\ldots+(-1)^{N} b_{N}\right) .
\end{aligned}
$$

Отсюда, согласно формулам (9), (10) и (12), следует:

$$
\begin{aligned}
& \left(a_{0}-a_{1}+-\ldots+(-1)^{N} a_{N}\right)^{2}=A_{0}, \\
& \left(b_{0}-b_{1}+-\ldots+(-1)^{N} b_{N}\right)^{2}=B_{0}=1-A_{0} .
\end{aligned}
$$

Эти соотношения тоже выполняются тождественно, независимо от азимутов пластинок.

Теперь потребуем, чтобы при $\cos \gamma=0$ лежал центр полосы пропускания с $I_{N}=1$, т. е. чтобы азимуты, согласно формулам (17) и (18), удовлетворяли условию

$$
\begin{aligned}
& \cos \left(2 \varphi_{N}-2 \varphi_{N-1}+-\ldots-(-1)^{N} 2 \varphi_{1}\right)=0 \\
& \left|\sin \left(2 \varphi_{N}-2 \varphi_{N-1}+\ldots \ldots-(-1)^{N} 2 \varphi_{1}\right)\right|=1
\end{aligned}
$$

Тогда, в силу тех же формул,

и

$$
a_{0}-a_{1}+-\ldots+(-1)^{N} a_{N}=1
$$

$$
b_{0}-b_{1}+-\ldots+(-1)^{N} b_{N}=0 \text {. }
$$

Отметим, что в правой части формулы (21) можно было бы написать -1. Мы выбрали +1 произвольно, так как, согласно формулам (3) и (7), изменение знаков всех $a_{k}$ равносильно изменению знаков всех азимутов, а это изменение несущественно. Наш выбор позволяет переписать вторую формулу (20) в виде:

$$
\sin \left(2 \varphi_{N}-2 \varphi_{N-1}+-\ldots-(-1)^{N} 2 \varphi_{1}\right)=(-1)^{N} .
$$

Подчеркнем, что формулы (21) и (22), в отличие от сходных формул (14), (15) и (19), не являются тождественными равенствами, но представляют собой условие, чтобы полоса пропускания находилась при $\cos \gamma=0$. Согласно формулам (19), при этом условии $A_{0}=1$ и $B_{0}=0$, так что формула (13) принимает вид:

$$
I_{N}(\cos \gamma)=1+A_{1} \cos ^{2} \gamma+\ldots+A_{N} \cos ^{2 N} \gamma .
$$


Далее рассмотрим связь между коэффициентами $a_{k}$ и $b_{k} \quad(k=$ $=0,1, \ldots, N)$ и азимутами. Связь эта определяется формулами (3), $(4)$, (7) и (8), но соответствующие выражения очень громоздки, так как содержат, вообще говоря, много членов. Простые выражения получаются только для $a_{0}, b_{0}, a_{N}$ и $b_{N}$. С учетом второй формулы (6) имеем:

$$
\begin{aligned}
& a_{0}=-\cos \varphi_{N} \cos \varphi_{N, N-1} \ldots \cos \varphi_{21} \sin \varphi_{1}, \\
& b_{0}=\sin \varphi_{N} \cos \varphi_{N, N-1} \ldots \cos \varphi_{21} \sin \varphi_{1}
\end{aligned}
$$

и

$$
\begin{aligned}
& a_{N}=\sin \varphi_{N} \cos \varphi_{N, N-1} \ldots \cos \varphi_{1}, \\
& b_{N}=\cos \varphi_{N} \cos \varphi_{N, N-1} \ldots \cos \varphi_{1} .
\end{aligned}
$$

Из этих формул следует:

$$
\tan \varphi_{1}=b_{0} / a_{N}=-a_{0} / b_{N}
$$

и

а также

$$
\tan \varphi_{N}=a_{N} / b_{N}=-b_{0} / a_{0},
$$

$$
a_{0} a_{N}+b_{0} b_{N}=0 .
$$

Наконец, нам понадобятся еще рекуррентные формулы для матрицы $F$ и ее элементов, а также для коэффициентов $a_{k}$ и $b_{k}$. Наряду с полным фильтром из $N$ пластинок будем рассматривать «неоконченные» фильтры, содержащие вместо $N$ пластинок только первые $m$ пластинок $(m=1,2, \ldots, N-1)$. Обозначим матрицу неоконченного фильтра, состоящего из $m$ пластинок, через $F^{(m)}$, а матрицу полного фильтра через $F^{(N)} \equiv F$. Согласно формулам $(7),(11)$ и (13) статьи $\left[{ }^{1}\right]$ имеет место рекуррентная формула

$$
F^{(m-1)}=\left(\begin{array}{cc}
\cos \gamma-i \sin \gamma \cos 2 \varphi_{m} & -i \sin \gamma \sin 2 \varphi_{m} \\
-i \sin \gamma \sin 2 \varphi_{m} & \cos \gamma+i \sin \gamma \cos 2 \varphi_{m}
\end{array}\right) F^{(m)} .
$$

Отсюда

$$
\begin{aligned}
& F_{21}^{(m-1)}=\cos \psi F_{21}^{(m)}+i \sin \gamma\left(F_{21}^{(m)} \cos 2 \varphi_{m}-F_{11}^{(m)} \sin 2 \varphi_{m}\right), \\
& F_{11}^{(m-1)}=\cos \gamma F_{11}^{(m)}-i \sin \gamma\left(F_{11}^{(m)} \cos 2 \varphi_{m}+F_{21}^{(m)} \sin 2 \varphi_{m}\right) .
\end{aligned}
$$

Для элементов каждого неоконченного фильтра можно написать формулы, аналогичные (7) и (8):

$$
\begin{aligned}
& F_{21}^{(m)}=e^{-i m \gamma}\left(a_{0}^{(m)}+a_{1}^{(m)} e^{2 i \gamma}+\ldots+a_{m}^{(m)} e^{2 i m \gamma}\right), \\
& F_{11}^{(m)}=e^{-i m \gamma}\left(b_{0}^{(m)}+b_{1}^{(m)} e^{2 i \gamma}+\ldots+b_{m}^{(m)} e^{2 i m \gamma}\right),
\end{aligned}
$$

где все коэффициенты $a_{k}^{(m)}$ и $b_{k}^{(m)}(k=1,2, \ldots, m)$ зависят от азимутов первых $m$ пластинок, подобно тому, как коэффициенты $a_{k} \equiv a_{k}^{(N)}$ и $b_{k} \equiv b_{k}^{(N)}$ зависят от азимутов всех $N$ пластинок. В частности, тождественные соотношения, аналогичные формулам (14) и (15), выполняются и для любого неоконченного фильтра:

$$
\begin{aligned}
& a_{0}^{(m)}+a_{1}^{(m)}+\ldots+a_{m}^{(m)}=0, \\
& b_{0}^{(m)}+b_{1}^{(m)}+\ldots+b_{m}^{(m)}=1 .
\end{aligned}
$$

Наоборот, соотношения типа формул (22) для неоконченных фильтров не имеют места. Но формулы, аналогичные формулам (25) и (26), опять справедливы для всех неоконченных фильтров: 


$$
\begin{aligned}
& a_{0}^{(m)}=-\cos \varphi_{m} \cos \varphi_{m, m-1} \ldots \cos \varphi_{21} \sin \varphi_{1}, \\
& b_{0}^{(m)}=\sin \varphi_{m} \cos \varphi_{m, m-1} \ldots \cos \varphi_{21} \sin \varphi_{1}
\end{aligned}
$$

И

$$
\begin{aligned}
a_{m}^{(m)} & =\sin \varphi_{m} \cos \varphi_{m, m-1} \ldots \cos \varphi_{1}, \\
b_{m}^{(m)} & =\cos \varphi_{m} \cos \varphi_{m, m-1} \ldots \cos \varphi_{1},
\end{aligned}
$$

откуда, подобно формулам (27)-(29), вытекает:

$$
\begin{aligned}
& \tan \varphi_{1}=b_{0}^{(m)} / a_{m}^{(m)}=-a_{0}^{(m)} / b_{m}^{(m)}, \\
& \tan \varphi_{m}=\mathrm{a}_{m}^{(m)} / b_{m}^{(m)}=-b_{0}^{(m)} / a_{0}^{(m)}
\end{aligned}
$$

и

$$
a_{0}^{(m)} a_{m}^{(m)}+b_{0}^{(m)} b_{m}^{(m)}=0 .
$$

Из рекуррентных формул (31) с помощью выражений (32) легко выводятся простые рекуррентные формулы и для коэффициентов $a_{k}^{(m)}$ и $b_{k}^{(m)}$. Подставляя в формулы (31) выражения матричных элементов $F_{11}^{(m)}, F_{21}^{(m)}, F_{11}^{(m-1)}$ и $F_{21}^{(m-1)}$ из формул (32) и учитывая равенства (37), находим:

$$
\begin{array}{r}
a_{k}^{(m-1)}=a_{k}^{(m)} \cos ^{2} \varphi_{m}+a_{k+1}^{(m)} \sin ^{2} \varphi_{m}+\left(b_{k+1}^{(m)}-b_{k}^{(m)}\right) \sin \varphi_{m} \cos \varphi_{m}, \\
b_{k}^{(m-1)}=b_{k}^{(m)} \sin ^{2} \varphi_{m}+b_{k+1}^{(m)} \cos ^{2} \varphi_{m}+\left(a_{k+1}^{(m)}-a_{k}^{(m)}\right) \sin \varphi_{m} \cos \varphi_{m}, \\
m=N, N-1, \ldots, 2, \\
k=0,1, \ldots, m-1 .
\end{array}
$$

\section{Синтез}

Выше мы уже положили, что в центре полосы пропускания, где $I_{N}=1$, должно быть $\cos \gamma=0$. Условие минимума усредненного по спектру коэффициента пропускания вне полосы пропускания приводит к системе линейных уравнений для коэффициентов $a_{0}, a_{1}, \ldots, a_{N}$. Первый этап синтеза и состоит в определении значений этих коэффициентов. Второй этап заключается в нахождении азимутов.

Итак, положим

$$
\bar{I}_{N}=\frac{1}{1-f} \int_{f}^{1} I_{N}(\cos \gamma) d \cos \gamma=\min ,
$$

где $f$ выбирается произвольно в рамках компромисса: если хотим уменьшить ширину полосы пропускания, то $f$ следует выбрать ближе к нулю, но тогда $I_{N}$ будет в среднем вне полосы пропускания больше; наоборот, если хотим сильнее подавить $I_{N}$ вне полосы пропускания, то $f$ следует увеличить, а это приведет к уширению полосы пропускания. Как убедимся в дальнейшем на примерах, обоим требованиям можно удовлетворить вполне приемлемым образом.

Подставляя в формулу (40), согласно формулам (7) и (13),

$$
I_{N}(\cos \gamma)=\left|a_{0}+a_{1} e^{2 i \gamma}+\ldots+a_{N} e^{2 i N \gamma}\right|^{2}
$$

и обозначая 


$$
\frac{1}{1-f} \int_{f}^{1} \cos 2 k \gamma d \cos \gamma=C_{k}, \quad k=1,2, \ldots, N
$$

находим:

$$
\begin{aligned}
\bar{I}_{N}= & a_{0}^{2}+a_{1}^{2}+\ldots+a_{N}^{2}+2 C_{1}\left(a_{0} a_{1}+a_{1} a_{2}+\ldots+a_{N-1} a_{N}\right)+ \\
& +2 C_{2}\left(a_{0} a_{2}+a_{1} a_{3}+\ldots+a_{N-2} a_{N}\right)+\ldots+2 C_{N} a_{0} a_{N} .
\end{aligned}
$$

Минимизируя $\bar{I}_{N}$, получаем с учетом уравнений (14) и (21) $N-1$ линейных уравнений для $a_{0}, a_{1}, \ldots, a_{N}$, которые, вместе с теми же уравнениями (14) и (21), определяют все эти коэффициенты. Решение уравнений облегчается наличием соотношений

$$
a_{k}=(-1)^{N} a_{N-k}, \quad k=0,1, \ldots, N,
$$

вытекающих из инвариантности уравнений (14) и (21) и выражения $I_{N}$ в формуле (43) относительно замены $a_{k} \rightleftarrows(-1)^{N} a_{N-k}(k=0$, $1, \ldots, N)$.

Переходим ко второму этапу синтеза. Коэффициенты $a_{0}, a_{1}, \ldots, a_{N}$ зависят от азимутов пластинок, и задача состоит в нахождении азимутов по известным значениям этих коэффициентов. Как было уже отмечено, связь между теми и другими величинами весьма громоздка. Поэтому эту задачу следует решать несколько окольным путем, состоящим из двух отдельных шагов.

Первый шаг состоит в определении коэффициентов $b_{0}, b_{1}, \ldots, b_{N}$. Для этого нужно решить вытекающее из формул (8) и (10) уравнение

$$
B_{0}+B_{1} \cos ^{2} \gamma+\ldots+B_{N} \cos ^{2 N} \gamma=\left|b_{0}+b_{1} e^{2 i \gamma}+\ldots+b_{N} e^{2 i N \gamma}\right|^{2},
$$

являющееся тождественным относительно $\gamma$ соотношением. Коэффициенты $B_{0}, B_{1}, \ldots, B_{N}$ определяются согласно формулам (12) по $A_{0}$, $A_{1}, \ldots, A_{N}$, а эти последние вычисляются согласно формулам (7) и (9) из соотношения

$$
A_{0}+A_{1} \cos ^{2} \gamma+\ldots+A_{N} \cos ^{2 N} \gamma=\left|a_{0}+a_{1} e^{2 i \gamma}+\ldots+a_{N} e^{2 i N \gamma}\right|^{2} .
$$

Напомним, кстати, что, поскольку при определении $a_{0}, a_{1}, \ldots, a_{N}$ было использовано уравнение $(21)$, то $A_{0}=1$ и $B_{0}=0$. Формулы (45) и (46) имеют одинаковый вид, но используются в противоположных направлениях. В формуле (46) $a_{0}, a_{1}, \ldots, a_{N}$ известны, и по ним вычисляются $A_{0}, A_{1}, \ldots, A_{N}$, что не представляет никаких трудностей. В формуле (45), наоборот, $B_{0}, B_{1}, \ldots, B_{N}$ известны, а $b_{0}, b_{1}, \ldots, b_{N}$ требуется найти. Эта задача не столь проста. Можно было бы, правда, написать $N+1$ квадратных уравнений для величин $b_{0}, b_{1}, \ldots, b_{N}$, приравнивая коэффициенты одинаковых степеней $\cos \gamma$ в равенстве (45) слева и справа. Однако прямое решение этой системы было бы очень громоздко. Поэтому предложим в нижеследующем иной способ.

Составим уравнение $N$-й степени

$$
B_{N} z^{N}+B_{N-1} z^{N-1}+\ldots+B_{1} z+B_{0}=0 .
$$

Решив его, получим $N$ корней, один из которых, так как $B_{0}=0$, равен нулю. Обозначим корни через $z_{0}=0, z_{1}, z_{2}, \ldots, z_{N-1}$. Положим затем

$$
u_{k}=2 z_{k}-1 \pm 2 i \sqrt{z_{k}\left(1-z_{k}\right)}, \quad k=0,1, \ldots, N-1
$$

Смысл этого в том, что если $z_{k}=\cos ^{2} \Theta_{k}$, то $u_{k}^{ \pm}=e^{ \pm 2 i \Theta_{k}}$. Таким обра- 
зом, левая часть равенства (45) обращается при $\gamma=\Theta_{0}, \Theta_{1}, \ldots, \Theta_{N-1}$ в нуль; следовательно, правая часть должна при $e^{2 i \gamma}=u_{0}, u_{1}, \ldots, u_{N-1}$ тоже обращаться в нуль. А это значит, что один из двух множителей (безразлично какой), образующих правую часть, обращается в нуль. Следовательно, мы получим $N$ линейных уравнений для $b_{0}, b_{1}, \ldots, b_{N}$ :

$$
\begin{gathered}
b_{0}-b_{1}+\ldots+(-1)^{N} b_{N}=0, \\
b_{0}+b_{1} u_{1}+\ldots+b_{N} u_{1}^{N}=0, \\
b_{0}+b_{1} u_{2}+\ldots+b_{N} u_{2}^{N}=0 \\
\vdots \\
b_{0}+b_{1} u_{N-1}+\ldots+b_{N} u_{N-1}^{N}=0
\end{gathered}
$$

(отметим, что первое из этих уравнений совпадает с уже известным уравнением (22)). Каждое из уравнений системы (49) (кроме первого) может быть написано в двух вариантах, так как $u_{k}$ может в нем означать $u_{k}^{+^{-*}}$ или $u_{k}^{-}$(чему соответствует равенство нулю того или другого множителя правой части (45)). Поэтому, казалось бы, мы имеем $2^{N-1}$ различных вариантов системы (49). На самом деле число вариантов меньше. Во-первых, необходимо учесть, что все $b_{k}$ должны быть вещественны; во-вторых, может случиться, что среди корней уравнения (47) есть кратные.

Прежде всего убедимся, что уравнение (47) в интервале $0<z \leqslant 1$ вещественных корней не имеет. Әто вытекает из того, что в противном случае при некотором вещественном значении $\gamma$, неравном $\arccos 0$, было бы $I_{N}(\cos \gamma)=F_{21} F_{21}^{*}=1-F_{11} F_{11}^{*}=1$. Но это противоречит условию (40), согласно которому $I_{N}=1$ только при $\cos \gamma=0$. Из отсутствия меньших единицы положительных вещественных корней вытекает, что вещественным корням $z_{k}$ соответствуют вещественные значения $u_{k}^{ \pm}$, а комплексным корням $z_{k}$ - комплексные значения $u_{k}^{ \pm}$,

Положим теперь сначала, что уравнение (47) кратных корней не имеет. Тогда все уравнения системы (49) независимы друг от друга. Присоединив к этой системе уравнение (15), получим систему $N+1$ линейных уравнений, которая и определяет все коэффициенты $b_{0}$, $b_{1}, \ldots, b_{N}$. Найдем число различных вариантов этого решения. Пусть уравнение (47) имеет $r$ ненулевых вещественных корней и $q$ пар комплексно-сопряженных. Каждому ненулевому вещественному корню соответствуют два вещественных значения $u^{ \pm}$; следовательно, уравнение системы (49), соответствующее этому корню, будет иметь вещественные коэффициенты и его можно написать в двух независимых вариантах. Всего $r$ таких корней дадут $2^{r}$ вариантов. Далее, если $z$ и $z^{*}-$ пара комплексно-сопряженных корней, то $u^{+}(z)$ и $u^{-}\left(z^{*}\right)$ тоже комплексносопряженны. Два уравнения системы (49), составленные с $u^{+}(z)$ и $u^{-}\left(z^{*}\right)$, будут иметь, следовательно, комплексно-сопряженные коэффициенты; поэтому они равносильны двум уравнениям с вещественными коэффициентами. В другом возможном варианте используются $u$ - $(z)$ и $u^{+}\left(z^{*}\right)$. Таким образом, каждой паре комплексно-сопряженных корней соответствуют два варианта, а всем $q$ парам - $2^{q}$ вариантов. Следовательно, полное число вариантов системы (49), дающих вещественные значения $b_{0}, b_{1}, \ldots, b_{N}$, равно $2^{r+q}$. Нулевой корень $z_{0}=0$ число вариантов не увеличивает, так как ему соответствует только одно значение $u_{0}=-1$.

Следует, однако, отметить, что, как вытекает из инвариантности пра- 
вой части уравнения (45) относительно замены $b_{k} \rightleftarrows b_{N-k}$, каждому решению этого уравнения соответствует другое, получающееся из него путем той же замены. Такие решения входят в число $2^{r+q}$ как различные (что видно хотя бы из равенства $\left.u_{k}^{+} u_{k}^{-}=1\right)$. А так как различие это несущественно (что будет показано в следующем разделе), то число существенно различных решений равно $2^{r+q-1}$.

Перейдем к случаю, когда уравнение (47) имеет кратные корни. Покажем, что число существенно различных решений равно и в этом случае $2^{r+q-1}$, только под $r$ следует понимать число р азличны х ненулевых корней уравнения (47), а под $q$ - число р азличных пар комплексно-сопряженных корней. Вместе с тем покажем, каким образом следует модифицировать систему (49), чтобы число независимых уравнений осталось в ней равным $N$. Для этого нужно, чтобы каждому кратному корню соответствовало равное кратности число уравнений.

Пусть, например, z двукратный корень. Мы можем считать его совокупностью двух неравных, но бесконечно близких корней, $z$ и $z+d z$, с последующим стремлением $d z$ к нулю. Соответственно, из формулы (48) получим два значения, $u$ и $u+d u$, которые дадут два уравнения системы (49):

и

$$
b_{0}+b_{1} u+b_{2} u^{2}+\ldots+b_{N} u^{N}=0
$$

$$
b_{0}+b_{1}(u+d u)+b_{2}\left(u^{2}+2 u d u\right)+\ldots+b_{N}\left(u^{N}+N u^{N-1} d u\right)=0 .
$$

Вычитая первое из второго и сокращая на $d u$, находим

$$
b_{1}+2 b_{2} u+3 b_{3} u^{2}+\ldots+N b_{N} u^{N-1}=0 .
$$

Это уравнение вместе с первым из двух предыдущих и образует пару уравнений системы (49), соответствующую парному корню. Аналогичное рассуждение приводит легко к выводу, что $j$-кратному корню соответствуют следующие $j$ уравнений:

$$
\begin{gathered}
b_{0}+b_{1} u+\ldots+b_{N} u^{N}=0, \\
\frac{d}{d u}\left(b_{0}+b_{1} u+\ldots+b_{N} u^{N}\right)=0, \\
\vdots \\
\frac{d^{j-1}}{d u^{j-1}}\left(b_{0}+b_{1} u+\ldots+b_{N} u^{N}\right)=0 .
\end{gathered}
$$

Таким образом, число линейных уравнений, определяющих коэффициенты $b_{0}, b_{1}, \ldots, b_{N}$, остается при наличии кратных корней по-прежнему равным $N+1$.

Переходя к вопросу о числе возможных вариантов этой системы уравнений, видим, что каждая группа уравнений $(50)$, соответствующая кратному корню, определяется полностью первым уравнением этой группы. А оно возможно в двух вариантах для каждого ненулевого вещественного корня и для каждой пары комплексных корней. Отсюда и вытекает, что число всех вариантов равно $2^{r+q-1}$, где $r-$ число р а 3 ли чных, не равных нулю вещественных корней и $q-$ число р аз л и чны х пар комплексно-сопряженных корней.

Итак, первый шаг второго этапа синтеза выполнен. Второй шаг, завершающий процедуру синтеза, состоит в определении азимутов $\varphi_{1}, \varphi_{2}, \ldots, \varphi_{N}$. Для этого имеем формулы (28) и (37) и рекуррентные формулы (39). Азимуты определяются по формулам (28) и (37) в порядке убывающих индексов, причем каждому применению формулы (37) предшествует применение рекуррентных формул (39). Вся проце- 
дура начинается с вычисления $\varphi_{N}$ по формуле (28). После этого вычисляются по формулам (39) коэффициенты $\underset{k}{a_{k}^{(N-1)}}$ и $b_{k}^{(N-1)} \quad(k=0$, $1, \ldots, N-1)$, которые дают по формуле (37) $\varphi_{N-1}$; затем цикл в том же порядке повторяется, пока не будет получен последний азимут $\varphi_{1}$. Тем самым синтез заканчивается. Во всех $2^{r+q-1}$ вариантах синтезированного фильтра коэффициент пропускания $I_{N}(\cos \gamma)$, согласно формуле (24), один и тот же.

\section{Дополнительные замечания к синтезу}

Вышеизложенная процедура вычисления оптимальных азимутов допускает разнообразный текущий и конечный контроль. Формулами конечного контроля являются формула (23) и соотношения

$$
\varphi_{k}=(-1)^{N+1} \varphi_{N+1-k}, \quad k=1,2, \ldots, N,
$$

которые докажем здесь методом полной индукции. Прежде всего, из формул (27), (28) и (44) вытекает, что

$$
\varphi_{1}=(-1)^{N+1} \varphi_{N},
$$

т. е. формула (51) верна при $k=1$. Чтобы распространить это соотношение на остальные азимуты, покажем, что если от данного фильтра, удовлетворяющего условию (44), отнимем его крайние пластинки, то для такого «урезанного» фильтра условие (44) тоже выполняется.

Прежде всего, как вытекает из формулы (7), условию (44) можно дать эквивалентное выражение

$$
F_{21}^{*}=(-1)^{N} F_{21} \text {. }
$$

Еще иначе можно то же написать в виде

$$
F^{\prime}=\sigma F \sigma,
$$

где

$$
\sigma=\left(\begin{array}{cc}
1 & 0 \\
0 & (-1)^{N+1}
\end{array}\right)
$$

a $F^{\prime}$ означает матрицу фильтра в случае падения света с обратной стороны. Чтобы убедиться в этом, воспользуемся формулами $(7),(11)$ и (13) статьи $\left[{ }^{1}\right]$, согласно которым

$$
F=L_{N} L_{N-1} \ldots L_{1}
$$

и

$$
L_{k}=\left(\begin{array}{cc}
\cos \gamma+i \sin \gamma \cos 2 \varphi_{k} & i \sin \gamma \sin 2 \varphi_{k} \\
i \sin \gamma \sin 2 \varphi_{k} & \cos \gamma-i \sin \gamma \cos 2 \varphi_{k}
\end{array}\right) .
$$

Из последней формулы видно, что $L_{k}^{-1}=L_{k}^{*} ;$ следовательно,

А так как

$$
F^{\prime}=L_{1} L_{2} \ldots L_{N}=F^{*-1} \text {. }
$$

$$
F=\left(\begin{array}{cc}
F_{11} & -F_{21}^{*} \\
F_{21} & F_{11}^{*}
\end{array}\right)
$$

то, выражая отсюда с учетом формулы (11) обратную матрицу, находим

$$
F^{\prime}=\left(\begin{array}{rr}
F_{11} & F_{21} \\
-F^{*} & F^{*}
\end{array}\right)
$$


С другой стороны,

$$
\sigma F \sigma=\left(\begin{array}{cc}
F_{11} & (-1)^{N} F_{21}^{*} \\
-(-1)^{N} F_{21} & F_{11}^{*}
\end{array}\right) .
$$

Сравнивая это выражение с формулой (59), видим, что равенство (54) выполняется тогда и только тогда, когда имеет место равенство (53), т. е. условие (44).

Теперь покажем, что выполнение равенства (54) для данного фильтра влечет за собой выполнение такого же равенства для «урезанного» фильтра. Для этого заметим, что, согласно формулам (52) и (57),

$$
\begin{aligned}
& L_{N}=\sigma L_{1} \sigma, \\
& L_{1}=\sigma L_{N} \sigma .
\end{aligned}
$$

Следовательно, $F^{\prime}=\sigma L_{N} \sigma L_{2} \ldots L_{N-1} \sigma L_{1} \sigma$; подставляя это выражение в формулу (54), находим

$$
L_{2} L_{3} \ldots L_{N-1}=\sigma\left(L_{N}^{-1} F L_{1}^{-1}\right) \sigma .
$$

Но $L_{N}^{-1} F L_{1}^{-1}$ есть, согласно формуле (56), матрица «урезанного» фильтра. Обозначив ее через $\bar{F}$, имеем

$$
\bar{F}^{\prime}=\sigma \bar{F} \sigma,
$$

что и требовалось показать. Следовательно, условие (44) выполняется для коэффициентов $\bar{a}_{k}$ «урезанного» фильтра, т. е.

$$
\bar{a}_{k}=(-1)^{N-2} \bar{a}_{N-2-k} .
$$

Но так как азимуты остались после удаления крайних пластинок неизменными (т. е. $\bar{\varphi}_{1}=\varphi_{2}$ и $\left.\bar{\varphi}_{N-2}=\varphi_{N-1}\right)$, то

$$
\varphi_{2}=(-1)^{N+1} \varphi_{N-1} .
$$

Тем самым формула (51) доказана.

Соотношения (51) не обязательно использовать как контрольные формулы, но можно сократить с их помощью объем вычислений, находя по изложенной в предыдущем разделе схеме не все азимуты, а только $\varphi_{N}, \varphi_{N-1}, \ldots, \varphi_{\left[\frac{N+1}{2}\right]}$, и определяя остальные по формуле (51). В таком случае для конечного контроля остается только формула (23).

Текущий контроль возможен благодаря многочисленным соотношениям простого вида между коэффициентами $a_{k}^{(m)}$ и $b_{k}^{(m)}$, вычисляемыми по формулам (39). Напишем несколько таких соотношений (во всех них, если не указано иначе, $m=N, N-1, \ldots, 1)$.

Прежде всего, для текущего контроля можно использовать формулы (33). Далее, замечая, что в формуле (36) левая часть не зависит от $m$, находим:

$$
\begin{aligned}
& a_{m_{1}}^{\left(m_{1}\right)} b_{0}^{\left(m_{2}\right)}-a_{m_{2}}^{\left(m_{2}\right)} b_{0}^{\left(m_{1}\right)}=0, \\
& a_{0}^{\left(m_{1}\right)} b_{m_{2}}^{\left(m_{2}\right)}-a_{0}^{\left(m_{2}\right)} b_{m_{1}}^{\left(m_{1}\right)}=0, \\
& a_{0}^{\left(m_{1}\right)} a_{m_{2}}^{\left(m_{2}\right)}+b_{0}^{\left(m_{2}\right)} b_{m_{1}}^{\left(m_{1}\right)}=0 .
\end{aligned}
$$

Последняя формула совпадает при $m_{1}=m_{2}$ с формулой (38). 
Еще ряд соотношений вытекает из формулы (11). Ее следствием, как мы видели, являются формулы (12). Но мы можем также переписать формулы (9) и (10) в виде:

$$
\begin{aligned}
& F_{21} F_{21}^{*}=P_{0}+P_{1} \cos 2 \gamma+\ldots+P_{N} \cos 2 N \gamma, \\
& F_{11} F_{11}^{*}=Q_{0}+Q_{1} \cos 2 \gamma+\ldots+Q_{N} \cos 2 N \gamma,
\end{aligned}
$$

откуда

$$
\begin{aligned}
& P_{0}+Q_{0}=1, \\
& P_{k}+Q_{k}=0, \quad k=1,2, \ldots, N .
\end{aligned}
$$

Выразив здесь $P$ и $Q$ согласно формулам (7) и (8) через $a$ и $b$ и заменив $N$ на $m$, получим $m+1$ соотношений, в том числе формулу (38) и еще, например,

$$
\begin{gathered}
\sum_{k=0}^{m}\left(b_{k}^{(m)^{2}}+a_{k}^{(m)^{2}}\right)=1, \\
a_{0}^{(m)} a_{m-1}^{(m)}+a_{1}^{(m)} a_{m}^{(m)}+b_{0}^{(m)} b_{m-1}^{(m)}+b_{1}^{(m)} b_{m}^{(m)}=0, \quad m>1
\end{gathered}
$$

и т. Д.

В заключение этого раздела покажем, что два симметричных решения системы уравнений (49), получающиеся одно из другого заменой $b_{k} \rightleftarrows b_{N-k}$, приводят к азимутам, переходящим друг в друга путем поворота на $90^{\circ}$ с предшествующим, в случае нечетного $N$, изменением знака. Такие решения следует, очевидно, считать одинаковыми, что мы и учли уже при подсчете числа возможных вариантов в предыдущем разделе.

Обозначим решения системы (49), о которых идет речь, через $b_{k}^{\prime}$ и $b_{k}^{\prime \prime}$, так что

$$
b_{k}^{\prime \prime}=b_{N-k}^{\prime}, \quad k=0,1, \ldots, N .
$$

Теми же верхними индексами ' и " будем обозначать все другие величины, относящиеся к тому или другому решению. Покажем, что

$$
\begin{array}{ll}
b_{k}^{(m) \prime \prime}=b_{m-k}^{(m) \prime}, & \\
a_{k}^{(m) \prime \prime}=(-1)^{N} a_{m-k}^{(m) \prime}, & \begin{array}{l}
m=N, N-1, \ldots, 1, \\
k
\end{array}=0,1, \ldots, m
\end{array}
$$

и

$$
\tan \varphi_{m}^{\prime \prime}=(-1)^{N+1} \tan ^{-1} \varphi_{m}^{\prime} .
$$

В самом деле, при $m=N$ формулы (74) верны по условию (73) и согласно формуле (44), а формула (75) в силу формулы (28). А из формул (37) и (39) вытекает, что если формулы (74) и (75) верны для данного значения $m$, то они верны и для значения $m-1$. Следовательно, они верны для любого значения $m$.

Теперь из формулы (75) следует, что

$$
\varphi_{k}^{\prime \prime}=\frac{\pi}{2}+(-1)^{N} \varphi_{k}^{\prime}, \quad k=1,2, \ldots, N,
$$

причем, так как азимуты вообще определяются с точностью до л, эта формула симметрична относительно $\varphi_{k}^{\prime}$ и $\varphi_{k}^{\prime \prime}$. Итак, решения $\varphi_{k}^{\prime}$ и $\varphi_{k}^{\prime \prime}$ связаны друг с другом только операцией поворота на $90^{\circ}$ и, в случае нечетного $N$, переменой знака. Углы между азимутами в обоих решениях одни и те же. Поэтому оба нужно считать за одно. 


\section{Примеры}

В нижеследующем приведены результаты синтеза фильтра, состоящего из семи пластинок в двух случаях: при $f=1 / 3$ и при $f=0,4$ (см. формулу (40)). Все корни уравнения (47) (кроме $z_{0}=0$ ) в обоих случаях комплексные и различные; следовательно, оба фильтра получаются в четырех вариантах. В табл. 1 приведены значения коэффициентов $a_{k}$ и $b_{h}$, а в табл. 2 - значения азимутов. Верхнее число в каждом гнезде таблиц относится к первому фильтру $(f=1 / 3)$, нижнее - ко второму $(f=0,4)$.

На рис. 1 показаны «розы азимутов» для первого фильтра. У второго эта картина сходна. Формулы интенсивности (24) имеют следующий вид. Для первого фильтра

$$
\begin{aligned}
I_{7}(\cos \gamma)=1 & -17,1397 \cos ^{2} \gamma+117,275 \cos ^{4} \gamma-415,255 \cos ^{6} \gamma+ \\
& +828,016 \cos ^{8} \gamma-937,103 \cos ^{10} \gamma+561,308 \cos ^{12} \gamma- \\
& -138,101 \cos ^{14} \gamma,
\end{aligned}
$$

\begin{tabular}{|c|c|c|c|c|c|}
\hline \multirow[b]{2}{*}{$k$} & \multirow[b]{2}{*}{$a_{k}$} & \multicolumn{4}{|c|}{$b_{k}$} \\
\hline & & $\begin{array}{c}1 \text {-й } \\
\text { вариант }\end{array}$ & $\begin{array}{c}2 \text {-й } \\
\text { вариант }\end{array}$ & $\begin{array}{c}\text { 3-й } \\
\text { вариант }\end{array}$ & $\begin{array}{c}\text { 4-й } \\
\text { вариант }\end{array}$ \\
\hline 0 & $\begin{array}{l}0,091810 \\
0,072444\end{array}$ & $\begin{array}{l}0,176268 \\
0,145941\end{array}$ & $\begin{array}{l}0,046582 \\
0,034259\end{array}$ & $\begin{array}{l}0,010031 \\
0,006306\end{array}$ & $\begin{array}{l}0,037959 \\
0,026861\end{array}$ \\
\hline 1 & $\begin{array}{l}-0,103650 \\
-0,102910\end{array}$ & $\begin{array}{l}-0,495757 \\
-0,474446\end{array}$ & $\begin{array}{l}-0,070311 \\
-0,065076\end{array}$ & $\begin{array}{l}-0,026012 \\
-0,020155\end{array}$ & $\begin{array}{l}-0,147894 \\
-0,122158\end{array}$ \\
\hline 2 & $\begin{array}{l}0,146923 \\
0,152195\end{array}$ & $\begin{array}{l}0,108416 \\
0,177457\end{array}$ & $\begin{array}{r}-0,006533 \\
0,009852\end{array}$ & $\begin{array}{l}0,056190 \\
0,048052\end{array}$ & $\begin{array}{l}0,294896 \\
0,276711\end{array}$ \\
\hline 3 & $\begin{array}{l}-0,157617 \\
-0,172451\end{array}$ & $\begin{array}{l}0,688761 \\
0,710634\end{array}$ & $\begin{array}{l}-0,241353 \\
-0,222872\end{array}$ & $\begin{array}{l}-0,098030 \\
-0,091213\end{array}$ & $\begin{array}{l}-0,260126 \\
-0,292131\end{array}$ \\
\hline 4 & $\begin{array}{l}0,157617 \\
0,172451\end{array}$ & $\begin{array}{l}0,188797 \\
0,161864\end{array}$ & $\begin{array}{l}0,595399 \\
0,600125\end{array}$ & $\begin{array}{l}0,152209 \\
0,149977\end{array}$ & $\begin{array}{l}-0,196635 \\
-0,137013\end{array}$ \\
\hline 5 & $\begin{array}{l}-0,146923 \\
-0,152195\end{array}$ & $\begin{array}{l}0,259177 \\
0,227852\end{array}$ & $\begin{array}{l}0,630714 \\
0,634758\end{array}$ & $\begin{array}{l}-0,216226 \\
-0,220930\end{array}$ & $\begin{array}{l}0,685962 \\
0,718912\end{array}$ \\
\hline 6 & $\begin{array}{l}0,103650 \\
0,102910\end{array}$ & $\begin{array}{l}0,026520 \\
0,014739\end{array}$ & $\begin{array}{l}-0,135449 \\
-0,144235\end{array}$ & $\begin{array}{l}0,281570 \\
0,295665\end{array}$ & $\begin{array}{l}0,363780 \\
0,333441\end{array}$ \\
\hline 7 & $\begin{array}{l}-0,091810 \\
-0,072444\end{array}$ & $\begin{array}{l}0,047819 \\
0,035961\end{array}$ & $\begin{array}{l}0,180950 \\
0,153190\end{array}$ & $\begin{array}{l}0,840268 \\
0,832298\end{array}$ & $\begin{array}{l}0,222057 \\
0,195378\end{array}$ \\
\hline
\end{tabular}

таблица 1

\begin{tabular}{|c|c|c|c|c|}
\hline \multirow{2}{*}{$k$} & \multicolumn{4}{|c|}{$\varphi_{k}$} \\
\hline & 1-й вариант & 2-й вариант & 3-й вариант & 4-й вариант \\
\hline $1 ; 7$ & $\begin{array}{l}-62^{\circ} 29^{\prime} 13^{\prime \prime} \\
-63^{\circ} 36^{\prime} 02^{\prime \prime}\end{array}$ & $\begin{array}{l}-26^{\circ} 54^{\prime} 07^{\prime \prime \prime} \\
-25^{\circ} 18^{\prime} 35^{\prime \prime}\end{array}$ & $\begin{array}{l}-6^{\circ} 14^{\prime} 06^{\prime \prime} \\
-4^{\circ} 58^{\prime} 30^{\prime \prime}\end{array}$ & $\begin{array}{l}-22^{\circ} 27^{\prime} 46^{\prime \prime} \\
-20^{\circ} 20^{\prime} 40^{\prime \prime}\end{array}$ \\
\hline $2 ; 6$ & $\begin{array}{l}-27^{\circ} 53^{\prime} 25^{\prime \prime} \\
-27^{\circ} 30^{\prime} 34^{\prime \prime}\end{array}$ & $\begin{array}{l}-18^{\circ} 10^{\prime} 31^{\prime \prime} \\
-14^{\circ} 49^{\prime} 18^{\prime \prime}\end{array}$ & $\begin{array}{l}2^{\circ} 44^{\prime} 53^{\prime \prime \prime} \\
3^{\circ} 44^{\prime} 48^{\prime \prime}\end{array}$ & $\begin{array}{l}21^{\circ} 52^{\prime} 23^{\prime \prime} \\
25^{\circ} 12^{\prime} 20^{\prime \prime}\end{array}$ \\
\hline $3 ; 5$ & $\begin{array}{l}26^{\circ} 57^{\prime} 30^{\prime \prime} \\
30^{\circ} 34^{\prime} 19^{\prime \prime}\end{array}$ & $\begin{array}{l}22^{\circ} 45^{\prime} 16^{\prime \prime \prime} \\
28^{\circ} 38^{\prime} 03^{\prime \prime}\end{array}$ & $\begin{array}{l}-11^{\circ} 00^{\prime} 09^{\prime \prime \prime} \\
-10^{\circ} 20^{\prime} 26^{\prime \prime}\end{array}$ & $\begin{array}{l}2^{\circ} 41^{\prime} 03^{\prime \prime} \\
2^{\circ} 49^{\prime} 19^{\prime \prime}\end{array}$ \\
\hline 4 & $\begin{array}{l}29^{\circ} 43^{\prime} 24^{\prime \prime} \\
33^{\circ} 57^{\prime} 41^{\prime \prime}\end{array}$ & $\begin{array}{l}73^{\circ} 03^{\prime} 20^{\prime \prime} \\
81^{\circ} 17^{\prime} 33^{\prime \prime}\end{array}$ & $\begin{array}{l}5^{\circ} 01^{\prime} 44^{\prime \prime} \\
6^{\circ} 52^{\prime} 32^{\prime \prime}\end{array}$ & $\begin{array}{l}-38^{\circ} 18^{\prime} 12^{\prime \prime} \\
-40^{\circ} 27^{\prime} 22^{\prime \prime}\end{array}$ \\
\hline
\end{tabular}

Таблища 2 

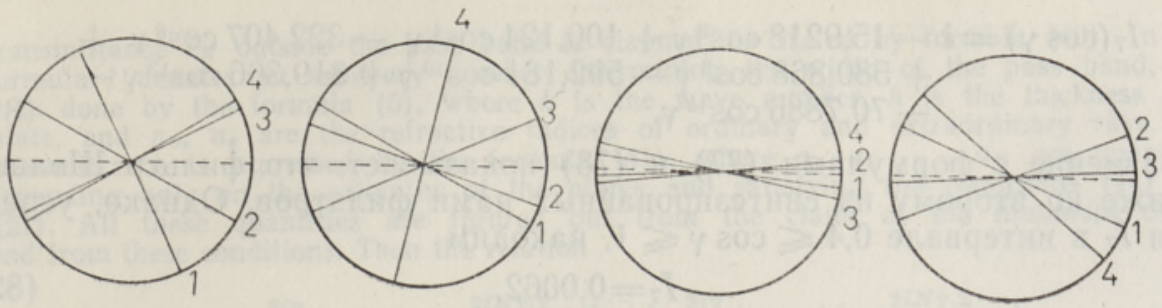

Рис. 1.

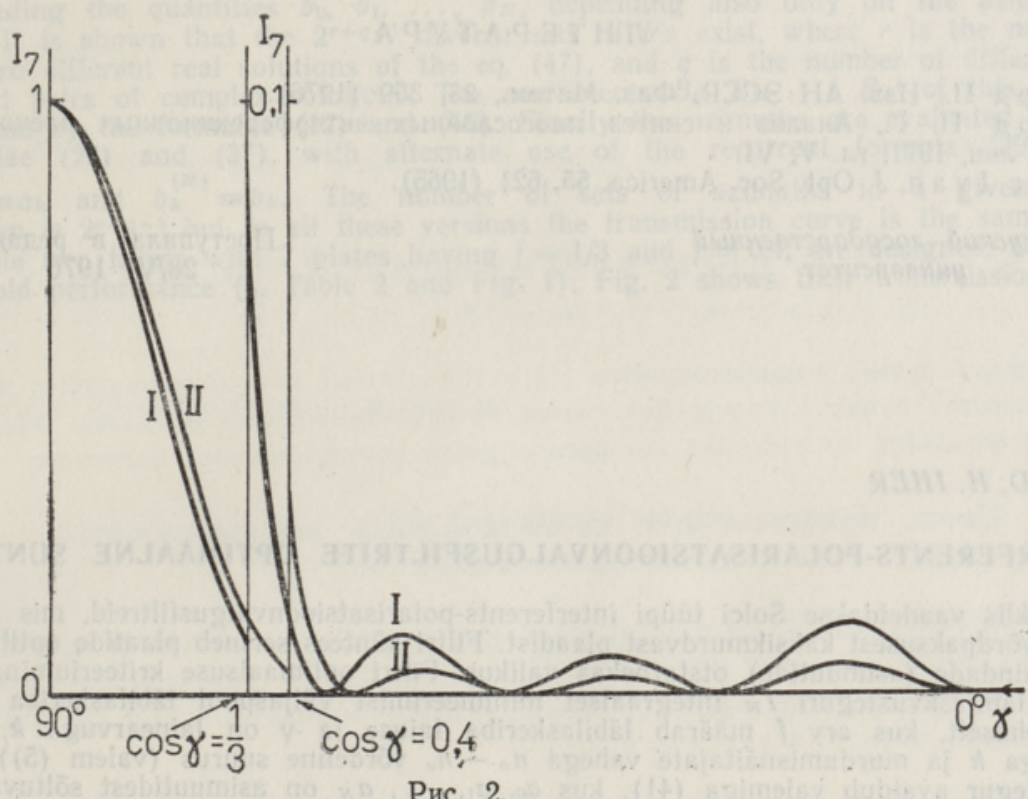

Рис. 2.

а для второго -

$$
\begin{aligned}
I_{7}(\cos \gamma)=1 & -15,3294 \cos ^{2} \gamma+95,4306 \cos ^{4} \gamma-312,928 \cos ^{6} \gamma+ \\
& +586,238 \cos ^{8} \gamma-630,447 \cos ^{10} \gamma+362,021 \cos ^{12} \gamma- \\
& -85,9851 \cos ^{14} \gamma .
\end{aligned}
$$

Вычисленные по этим формулам графики показаны на рис. 2.

Приведем еще усредненные (согласно формуле (40)) значения интенсивности вне полосы пропускания. Общая формула при $N=7$ имеет вид:

$$
\begin{gathered}
\bar{I}_{7, \min }=(1 / 2)\left(1-C_{1}\right)-\left(1-C_{1}+C_{3}-C_{4}\right) a_{0}+ \\
+\left(1-C_{1}-C_{2}+C_{3}\right) a_{1}-\left(1-C_{2}\right) a_{2} .
\end{gathered}
$$

Подставляя сюда значения $C_{1}, C_{2}, C_{3}, C_{4}$ из формулы (42) и $a_{0}, a_{1}, a_{2}$ из табл. 1, находим для наших двух фильтров:

$$
\bar{I}_{7, \min }=\left\{\begin{array}{l}
0,0084 \\
0,0036
\end{array}\right.
$$

Наконец, сравним наши результаты с фильтром Шольца $\left[{ }^{3}\right]$, в котором $\varphi_{1}=\varphi_{7}=4^{\circ} 30^{\prime}, \varphi_{2}=\varphi_{6}=-6^{\circ}, \varphi_{3}=\varphi_{5}=7^{\circ} 30^{\prime}$ и $\varphi_{4}=-9^{\circ}$. Формула $I_{7}$ дана в $\left[{ }^{3}\right]$ неточно. В уточненном виде она такова: 


$$
\begin{aligned}
I_{7}(\cos \gamma)=1 & -15,9218 \cos ^{2} \gamma+100,124 \cos ^{4} \gamma-322,407 \cos ^{6} \gamma+ \\
& +580,868 \cos ^{8} \gamma-592,181 \cos ^{10} \gamma+319,306 \cos ^{12} \gamma- \\
& -70,7886 \cos ^{14} \gamma .
\end{aligned}
$$

Сравнение с формулами (77) и (78) показывает, что фильтр Шольца ближе ко второму из синтезированных нами фильтров. Однако, усредняя $I_{7}$ в интервале $0,4 \leqslant \cos \gamma \leqslant 1$, находим

$$
\bar{I}_{7}=0,0062 \text {, }
$$

что значительно превышает минимум, равный 0,0036 .

\section{ЛИТЕРАТ У РА}

1. К а рд П., Изв. АН ЭССР, Физ. Матем., 25, 359 (1976).

2. К ард П. Г., Анализ и синтез многослойных интерференционных пленок, Таллин, 1971, гл. V, VI.

3. S o lc I v a n, J. Opt. Soc. America, 55, 621 (1965).

\section{Тартуский государственный} университет

\author{
Поступила в редакцию \\ 28/VI 1976
}

\section{P. KARD, H. IHER}

\section{INTERFERENTS-POLARISATSIOONVALGUSFILTRITE OPTIMAALNE SUNTEES}

Artiklis vaadeldakse Šolci tüüpi interferents-polarisatsioonvalgusfiltreid, mis koosnevad $N$ võrdpaksusest kaksikmurdvast plaadist. Filtri süntees seisneb plaatide optiliste telgede suundade (asimuutide) otstarbekas valikus. Filtri optimaalsuse kriteeriumina kasutatakse läbilaskvusteguri $I_{N}$ integraalset minimeerimist väljaspool läbilaskeriba valemi (40) kohaselt, kus arv $f$ määrab läbilaskeriba laiuse ja $\gamma$ on lainearvuga $k$, plaadi paksusega $h$ ja murdumisnäitajate vahega $n_{o}-n_{e}$ võrdeline suurus (valem (5)). Läbilaskvustegur avaldub valemiga (41), kus $a_{0}, a_{1}, \ldots, a_{N}$ on asimuutidest sõltuvad kordajad. Need määratakse miinimumitingimusest (40), arvestades ka seoseid (14) ja (21). Seejärel leitakse seosest

$$
\left|a_{0}+a_{1} e^{2 i \gamma}+\ldots+a_{N} e^{2 i N \gamma}\right|^{2}+\left|b_{0}+b_{1} e^{2 i \gamma}+\ldots+b_{N} e^{2 i N \gamma}\right|^{2}=1
$$

kordajad $b_{0}, b_{1}, \ldots, b_{N}$, mis samuti sõltuvad ainult asimuutidest. Näidatakse, et nendel kordajatel on $2^{r+q-1}$ väärtuste kogumit, kus $r$ on võrrandi (47) nullist ja üksteisest erinevate reaalsete lahendite arv ja $q$ on sama võrrandi erinevate kaaskomplekssete lahendipaaride arv; võrrandi kordajad $B_{0}, B_{1}, \ldots, B_{N}$ saadakse valemitest (12) ja (46). Asimuudid $\varphi_{N}, \varphi_{N-1}, \ldots, \varphi_{1}$ leitakse valemitest (28) ja (37) rekurrentsete valemite (39) kaasabil, kus $a_{k}^{(N)} \equiv a_{k}$ ja $b_{k}^{(N)} \equiv b_{k}$. Igal sünteesiprobłleemil on $2^{r+q-1}$ lahendust asimuutide osas, kuid läbilaskvuskõver on kõigis neis ühesugune. Näitena on sünteesitud 7 plaadist koosnevad filtrid, millel $f=1 / 3$ ja $f=0,4$, kumbki neljas variandis. Tulemused on esitatud joonistel 1 ja 2 ning tabelites 1 ja 2 .

\section{P. KARD, H. IHER}

\section{THE DESIGN OF A BIREFRINGENT CHAIN LIGHT-FILTER WITH OPTIMUM PERFORMANCE}

A method is developed for the design of a birefringent chain light-filter of the Solc type, consisting of a number, $N$, of birefringent plates of equal thickness. The design aims at the best choice of azimuths $\varphi_{m}(m=1,2, \ldots, N)$ of the plates. As a criterion for the optimum performance of the filter, the minimum of the averaged 
transmittance $I_{N}$ outside the pass band is claimed, as stated by formula (40). In this formula $f$ denotes an arbitrary number determining the width of the pass band, and $\mathcal{Y}$ is done by the formula (5), where $k$ is the wave number, $h$ is the thickness of a plate, and $n_{o}, n_{e}$ are the refractive indices of ordinary and extraordinary rays. The transmittance is expressed by the formula $(41)$, where $a_{0}, a_{1}, \ldots, a_{N}$ are quantities depending only on the azimuths of the plates and satisfying the conditions (14) and (21). All these quantities are figured out from the claim of the minimum of $I_{N}$ and from these conditions. Then the relation

$$
\left|a_{0}+a_{1} e^{2 i \nu}+\ldots+a_{N} e^{2 i N \gamma^{2}}\right|^{2}+\left|b_{0}+b_{1} e^{2 i \nu}+\ldots+b_{N} e^{2 i N \gamma}\right|^{2}=1
$$

for finding the quantities $b_{0}, b_{1}, \ldots, b_{N}$, depending also only on the azimuths, is used. It is shown that the $2^{r+q-1}$ diverse sets of $b$ 's exist, where $r$ is the number of non-zero different real solutions of the eq. (47), and $q$ is the number of different conjugated pairs of complex solutions. The coefficients $B_{0}, B_{1}, \ldots, B_{N}$ of this equation are done by the formulae (12) and (46). Finally the azimuths are evaluated from the formulae (28) and (37), with alternate use of the recurrent formula (39), where $a_{k}^{(N)} \equiv a_{k}$ and $b_{k}^{(N)} \equiv b_{k}$. The number of sets of azimuths in a given design problem is $2^{r+q-1}$ but in all these versions the transmission curve is the same. As an example two filters with 7 plates having $f=1 / 3$ and $f=0.4$, are designed. Both have four-fold performance (s. Table 2 and Fig. 1). Fig. 2 shows their transmission curves. 